\title{
Assessment of body composition in Wistar rat offspring by DXA in relation to prenatal and postnatal nutritional manipulation
}

\author{
Makarios Eleftheriades ${ }^{1,2}$, Homeira Vafaei ${ }^{3}$, Ismene Dontas ${ }^{4}$, George Vaggos ${ }^{5}$, Katerina Marinou ${ }^{6}$, Panagiota Pervanidou ${ }^{7}$, \\ Neil J. Sebire ${ }^{8}$, George P. Chrousos ${ }^{2,7}$ and Kypros H. Nicolaides ${ }^{9}$
}

BACKGROUND: This experimental study aims to investigate the impact of combinations of prenatal and postnatal food manipulation on body composition in rat offspring.

METHODS: On day 12 of gestation, 100 timed pregnant rats were randomized into two nutritional groups: standard laboratory and 50\% starved. Pups born to starved mothers were subdivided, based on birthweight (BiW), into fetal growth restricted (FGR) and non-FGR. Pups were born on day 21 , cross-fostered, then left undisturbed lactating until the 26th postnatal day when they underwent dual-energy X-ray absorptiometry (DXA) examination.

RESULTS: Prenatally control-fed animals had a significantly greater body weight at $26 \mathrm{~d}$ postnatally than the prenatally starved groups, irrespective of their postnatal diet $(P<0.001)$. Postnatal control diet was associated with significantly increased abdominal and total fat in non-FGR compared to FGR rats $(P<0.001)$. non-FGR/CONTROL rats showed higher values of abdominal fat than prenatally starved animals that were starved postnatally irrespective of their birth weight $(P<0.001)$. Postnatal control diet significantly increased total bone mineral content $(B M C)$, head $B M C$, head area, abdominal BMC in non-FGR compared to FGR rats $(P<0.001)$.

CONCLUSION: Interaction between prenatal and postnatal nutrition affects growth, abdominal adiposity, and bone accrual in Wistar rats' offspring at $26 \mathrm{~d}$ of life.

A range of genetic and environmental factors regulate growth and metabolism of individuals. Alterations in their interactions can produce adaptations that may permanently program their health and biological vulnerability to disease $(1-3)$. Obesity is associated with an increased risk of developing cardiometabolic disease contributing to an increase in cardiovascular morbidity and mortality worldwide. The risk is profoundly influenced by the pattern of fat distribution, and abdominal obesity is a key predictive factor of the metabolic syndrome. Additionally, visceral adiposity is associated with an unfavorable metabolic, dyslipidemic, and atherogenic obesity phenotype whereas abdominal subcutaneous adipose tissue is related to a more benign phenotype described by moderate association with inflammatory biomarkers and leptin. Moreover, abdominal subcutaneous adiposity has not been associated independently with dyslipidemia, insulin resistance, or atherosclerosis in obese individuals suggesting that abdominal fat distribution defines diverse obesity subphenotypes with heterogeneous metabolic and atherogenic risks (4).

Prenatal exposure to excessive or deficient nutrition alters adipocyte development (adipogenesis), permanently increasing the ability of adipose tissue to form new cells and to store lipids in existing adipocytes (lipogenesis). Adipogenesis is a late prenatal and early postnatal life phenomenon and is highly influenced by the nutritional environment at this time period. The number of adipocytes remains rather stable during adulthood, showing a very low turnover rate of adipose cells, providing evidence that events during both fetal and early postnatal life are vital for the overall development of adipose tissue $(5,6)$.

Body composition and musculoskeletal development begin in embryonic life, when bone and muscle develop from the mesodermal layer. Both bone mass and bone size increase throughout childhood, reaching their peak between 20 and $30 \mathrm{y}$ of age and at the end of puberty, respectively. Optimal bone growth achieved during childhood is not only critical for ensuring optimal development and protection from fractures during childhood, but is also associated with later changes in bone mineral density (BMD) that increase bone fragility and susceptibility to fractures in adulthood $(7,8)$.

Accumulating data from epidemiologic and experimental studies indicate that "early-life events" (prenatal and early postnatal) can initiate changes in gene expression, which determine not only the risk for postnatal disease but also an individual's

\footnotetext{
${ }^{1}$ Embryocare, Fetal Medicine Unit, Athens, Greece; ${ }^{2}$ First Department of Pediatrics, University of Athens School of Medicine, Aghia Sophia Children's Hospital, Athens, Greece; ${ }^{3}$ Department of Obstetrics and Gynecology, Faghihi Hospital, Shiraz University of Medical Sciences, Shiraz, Iran; ${ }^{4}$ Laboratory for Research of the Musculoskeletal System, School of Medicine, University of Athens, Athens, Greece; ${ }^{5}$ Department of Obstetrics and Gynecology, General Hospital of Arta, Arta, Greece; ${ }^{6} \mathrm{Greek}$ Ministry of Reconstruction of Production, Environment and Energy, Division of Animal Welfare, Athens, Greece; ${ }^{7}$ Childhood Obesity Clinic, First Department of Pediatrics, University of Athens, School of Medicine, Aghia Sophia Children's Hospital, Athens, Greece; ${ }^{8}$ Department of Paediatric Pathology, Great Ormond Street Hospital for Children, London, UK and UCL Institute of Child Health, London, UK; ${ }^{9}$ Harris Birthright Research Centre for Fetal Medicine, King's College Hospital, London, UK. Correspondence: Panagiota Pervanidou (ppervanid@med.uoa.gr; ppervanidou@gmail.com)

Received 13 September 2015; accepted 19 January 2016; advance online publication 11 May 2016. doi:10.1038/pr.2016.61
} 


\section{Articles Eleftheriades et al.}

response to the postnatal environment (9-12). Nutrition is one of the environmental variables with the widest range of effects on physical growth, metabolism, brain, adipose, and musculoskeletal development $(13,14)$.

Altered maternal nutrition may induce long-term metabolic consequences in offspring. However, the effects of maternal undernutrition during different developmental windows on body composition in offspring are not well defined. We investigated the effect of maternal undernutrition during pregnancy and/or lactation on postnatal growth, abdominal adiposity, and bone accrual in offspring. Animal studies commonly involve nutritional interventions of fetal or neonatal environment to investigate diseases with developmental origins. These interventions are mainly dietary and include global caloric restriction, dietary fat supplementation, or alterations of dietary protein content (15-17).

The majority of studies have not distinguished between the effects of maternal diet during pregnancy and those during the lactating period since the same diet continues postnatally until weaning. The contribution of maternal diet during suckling is also important as organ development and maturation continues after birth. Moreover, mismatch between fetal and postnatal environments, through manipulation of postnatal diet could be the basis of disease manifestation according to the predictive adaptive response hypothesis. The predictive adaptive response hypothesis refers to a form of developmental plasticity in which cues received in early life influence the development of a phenotype that is normally adapted to the environmental conditions of later life (18).

The aim of this study was to assess the effect of prenatal and postnatal food manipulation on weight status and body composition of the offspring during the lactating period. In particular, to examine the effects of prenatal starvation combined with postnatal food restriction or standard diet on growth and body composition of 26-d-old Wistar rats, compared to siblings of a standard nutritional perinatal environment. We hypothesized that mismatch of prenatal and postnatal nutritional status might have adverse effects on body composition in early postnatal life.

\section{RESULTS}

In total, 100 animals were studied. Table 1 shows the number of animals in each study group. The mean values for all DXA outcomes and comparisons between the different study groups

Table 1. Number of animals in each study group

\begin{tabular}{lccc}
\hline & No of animals & Males & Females \\
\hline A. CONTROL/CONTROL & 16 & 7 & 9 \\
B. FGR/CONTROL & 16 & 7 & 9 \\
C. non-FGR/CONTROL & 20 & 10 & 10 \\
D. FGR/FR & 21 & 10 & 11 \\
E. non-FGR/FR & 27 & 15 & 12 \\
Total & 100 & 49 & 51
\end{tabular}

FGR, fetal growth restricted; $F R$, food restricted. are presented in Table 2. The DXA output provides information about the following masses (in grams): fat, lean tissue, and bone mineral content $(\mathrm{BMC})$ of the total body and body regions.

CONTROL/CONTROL group had significantly greater values on weight, total (tot). Lean, tot. BMC, head (Hd) BMD, Hd BMC, Hd Area, abdominal (Abd). tissue mass (T.Mass), Abd. lean than FGR/CONTROL $(P<0.001)$, indicating that normal postnatal diet does not allow complete catch-up growth of FGR pups by day 26.

Furthermore, CONTROL/CONTROL group in comparison with non-FGR/CONTROL had significantly greater values on weight (g), tot. lean, Abd. BMD, Abd. BMC, Abd. area and $\mathrm{Abd}$. lean and significantly lower values on Abd. fat and tot. fat. Prenatally starved cases remained smaller than controls even if they reached normal body weight and received a normal postnatal diet, indicating that the effect of prenatal starvation persists to day 26.

In comparison with FGR/food restricted (FR) and non-FGR/ FR group, CONTROL/CONTROL group had significantly greater values on weight, tot. T.Mass, tot. lean, tot. BMC, Hd BMD, Hd BMC, Hd area, Abd. BMD, Abd. T.Mass, and Abd. lean.

FGR/CONTROL group had significantly lower values on weight, tot. fat, tot. lean, tot. BMC, Hd BMC, Hd Area, Abd. BMC, Abd. area, Abd. T.Mass, Abd. fat, and Abd. lean than the non-FGR/CONTROL group.

Furthermore, FGR/CONTROL group had significantly greater values on weight, tot. lean, tot. BMC, $\mathrm{Hd} \mathrm{BMD}, \mathrm{Hd}$ BMC, Hd area, Abd. BMD, Abd. T.Mass, and Abd. lean than FGR/FR and non-FGR/FR group.

non-FGR/CONTROL group had significantly greater values on weight, tot. T.Mass, tot. lean, tot. BMC, Hd BMD, Hd BMC, Hd area, Abd. BMD, Abd. BMC, Abd. area, Abd. T.Mass, Abd. fat, and Abd. lean than the FGR/FR and non-FGR/FR groups.

Weight was the only parameter that was greater in the nonFGR/FR group than the FGR/FR group.

$\%$ T. fat was lower in FGR/CONTROL than the non-FGR/ CONTROL, non-FGR/FR and FGR/FR groups and lower in CONTROL/CONTROL than the FGR/FR and non-FGR/FR groups.

Abd. fat was greater in non-FGR/CONTROL group than the FGR/CONTROL, FGR/FR and non-FGR/FR groups, while tot. Fat was greater in non-FGR/CONTROL group than the FGR/CONTROL group.

Multiple regression analyses (Table 3) for body weight showed lower values in the FR than the CONTROL group, in the FGR than the non-FGR group, and in females than in males.

A significant interaction effect of groups based on postnatal diet (CONTROL or FR) with FGR indicated that differences between FGR and non-FGR group are more evident in CONTROL group. When multiple regression analyses were conducted, with dependent variables all DEXA outcomes (Table 3), a significant group effect on almost all outcomes was noted. Specifically, for all DXA variables except for Abd. \%T.fat, the FR group had significantly lower values than the CONTROL group. Additionally, FGR animals had significantly 
Table 2. Mean values for all DXA outcomes and comparisons between different study groups

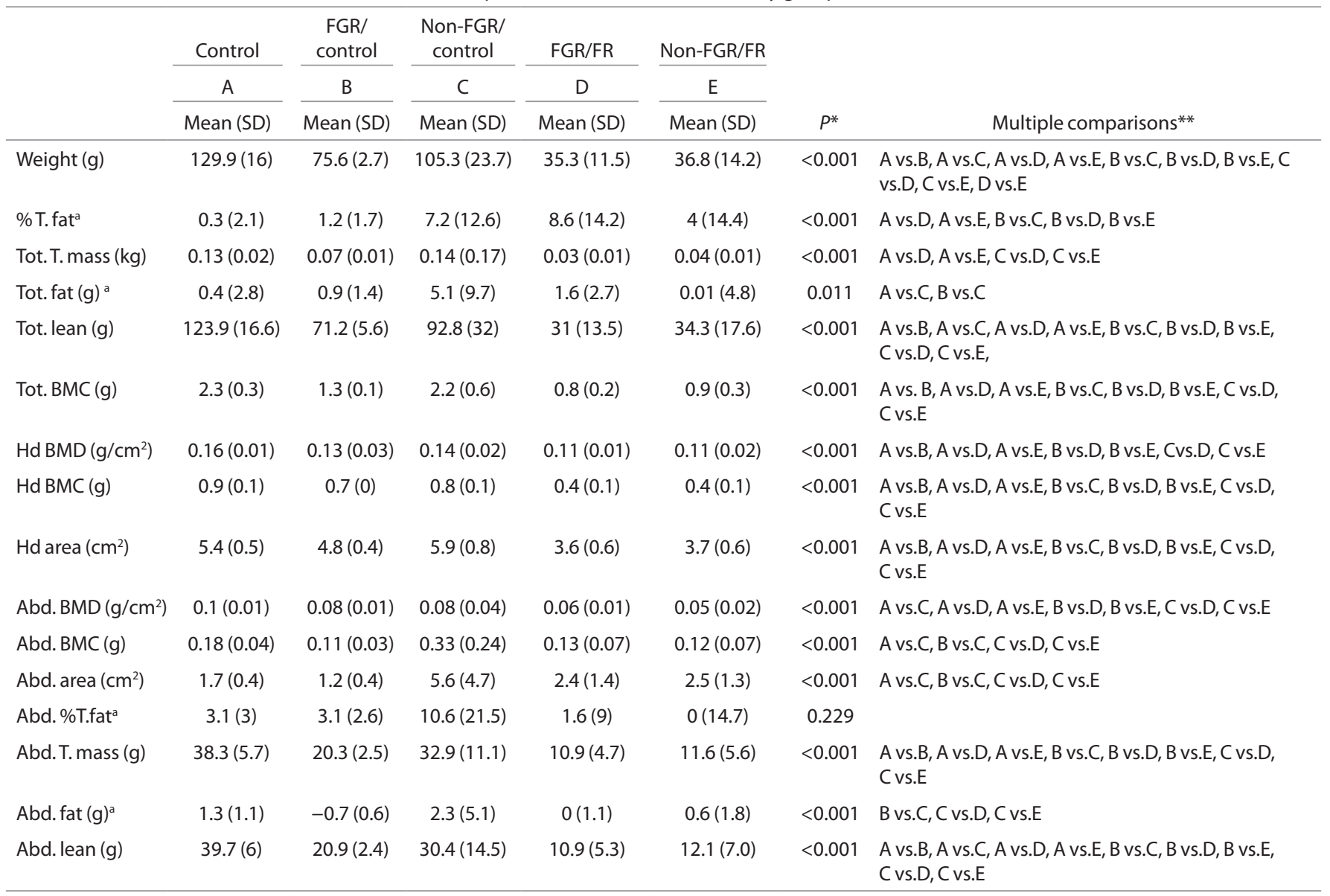

${ }^{a}$ Comparisons were based on log-transformed values.

${ }^{*} P$ value from ANOVA. ${ }^{*}$ Significant differences between groups after multiple comparisons with Bonferroni correction.

DXA, dual energy X-ray absorptiometry; FGR, fetal growth restricted; FR, food restricted.

lower values on all DXA outcomes except for $\% \mathrm{~T}$. fat, tot. T. mass, Hd BMD, Abd. BMD, and Abd. \%T.Fat. The effect of sex was significant on body weight, tot. lean, and Abd. lean, and females had significantly lower values. No significant interaction effect of sex with groups based on prenatal or postnatal diet was found. Furthermore, the interaction effect of groups based on postnatal diet (FR and CONTROL) with FGR was significant for tot. fat, tot. lean, tot. BMC, Hd BMC, Hd area, Abd. BMC, Abd. area, Abd. fat, and Abd. lean indicating that differences between FGR and non-FGR groups were more evident with the postnatal CONTROL diet.

\section{DISCUSSION}

DXA is an established method for body composition assessment that effectively characterizes lean and fat volume and bone mineral density both in rodents and humans (19-21). Our study demonstrated that a combination of specific prenatal and postnatal nutritional statuses produces distinct body composition profiles in the offspring that may have potential health implications in childhood or even later in adult life.

Regarding growth, maternal control-fed animals during pregnancy had a significantly greater body weight at $26 \mathrm{~d}$ postnatally than the prenatally starved groups irrespective of their postnatal food manipulation. The non-FGR/CONTROL animals had significantly greater body weight than the FGR/ CONTROL animals, the non-FGR/FR had significantly higher body weight than the FGR/FR subjects showing that starved Rats that were born $>-2$ SD maintained their higher body weight compared to starved rats that were born $<-2$ SD, at $26 \mathrm{~d}$ postnatally, irrespective of their postnatal food manipulation. Our data show that the effects of prenatal nutrition (either normal diet or starvation) on postnatal growth persist during the lactation period irrespective of the postnatal environment, indicating the importance of in utero adverse events on postnatal growth.

Mismatch between fetal and postnatal environments could lead to adult disease (22); thus, the manipulation of postnatal diet-by exposing the developing organism postnatally to the same amount of nutrition it was exposed prenatallycould theoretically prevent adverse metabolic consequences. As current medical interventions for FGR are mainly focused on the prevention of adverse perinatal complications (23), whereas postnatal therapeutics for FGR are lacking, it might be essential for FGR infants to implement both early nutritional 


\section{Articles | Eleftheriades et al.}

Table 3. Results from multiple linear regression models with dependent variables all DXA outcomes

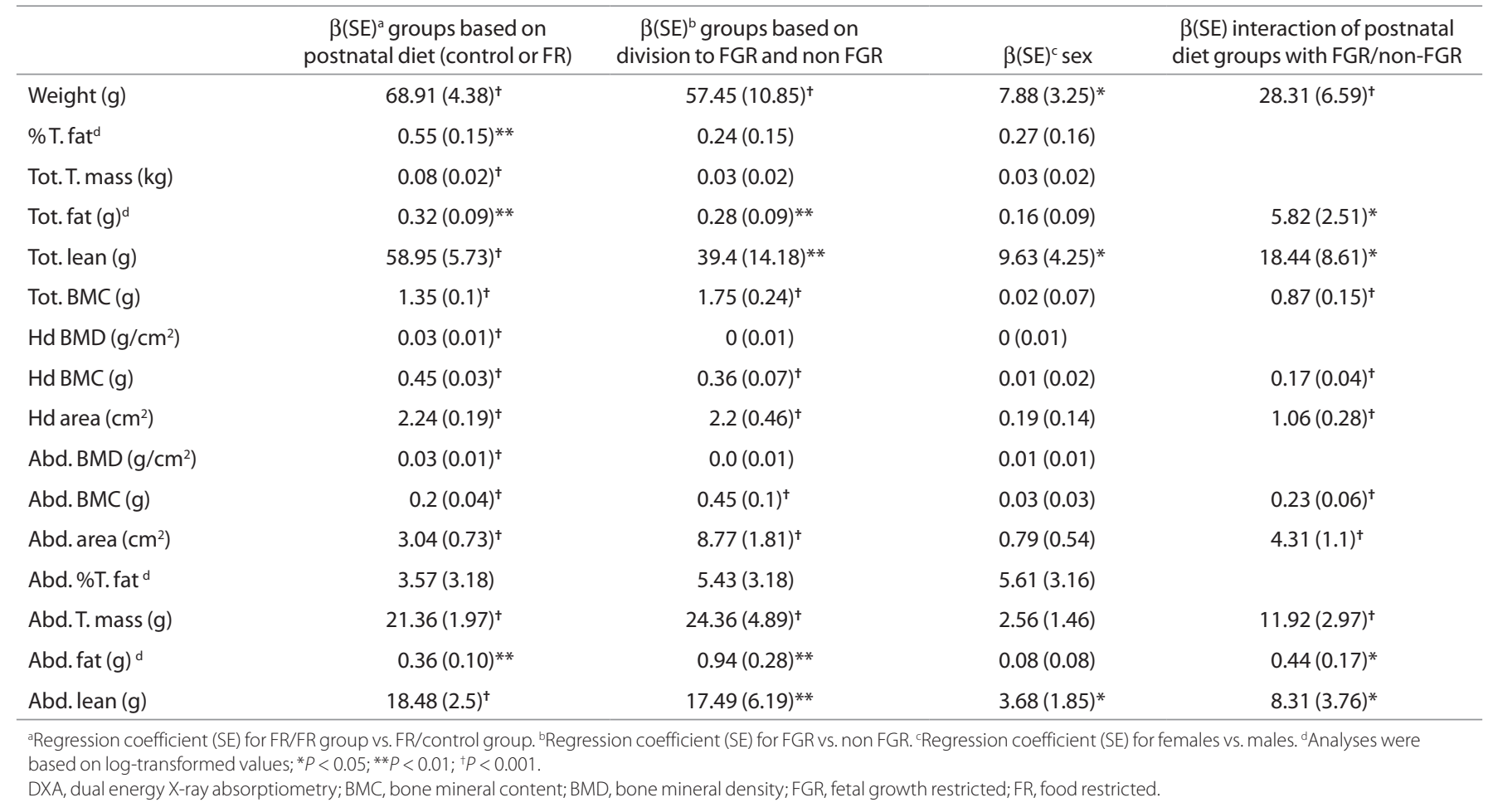

interventions such as the promotion of breastfeeding and the avoidance of overeating in order to catch-up and lifelong lifestyle interventions aiming at avoiding exposure to conditions of "plenty" (low fat diet consumption, regular body exercise).

Clinical studies have demonstrated a strong relation between abdominal fat and metabolic diseases, such as type 2 diabetes mellitus, dyslipidemia, and cardiovascular disease (24-26). Indeed, abdominal obesity is strongly associated with metabolic disorders in humans, and body composition analysis and fat distribution are important to study and understand the mechanisms involved in metabolic regulation. A strong relation between abdominal fat accumulation and metabolic alterations has been documented even in subjects with normal BMI (24-26).

In a recent study where DXA was used to assess abdominal obesity in an adolescent population, the strongest association was observed between abdominal obesity and insulin resistance, suggesting the key impact of abdominal obesity on the development of metabolic syndrome (27).

It has been postulated that the detrimental effects of visceral adipocytes on metabolism are due to their macrophage infiltration and proinflammatory cytokine production, which then influence liver metabolism and increase metabolic risk factors $(28,29)$. In a prospective cohort study regarding fetal and infant growth patterns and their association with total and abdominal fat distribution in childhood, growth during both fetal life and infancy affected childhood BMI, whereas only infant growth directly affected measured total body and abdominal fat. Interestingly, fetal growth deceleration followed by infant growth acceleration may lead to an adverse body fat distribution in childhood (30). Experimental studies have also established abdominal obesity as a potential biomarker for metabolic abnormalities (liver fat accumulation, insulin resistance/diabetes), similar to that described in clinical studies (31).

In our experimental model, prenatal food restriction had a significant impact on abdominal adiposity. Starved rats that were born $>-2 \mathrm{SD}$ and received standard diet postnatally showed greater values of Abd. fat and tot. fat. than starved animals that were born $<-2$ SD and that received standard diet postnatally, indicating that under standard postnatal nutrition, birth weight in prenatally starved rats may contribute significantly in adipose tissue accumulation. Starved rats that were born $>-2$ SD and received standard diet postnatally showed greater values of Abd. fat than prenatally starved animals which were starved postnatally, irrespective of their birthweight. Additionally, the same group of prenatally starved animals showed greater values of Abd. fat and tot. fat than controls, whereas postnatally starved groups showed no difference in Abd. fat and tot. fat compared to controls, supporting the concept of fetal and neonatal environmental mismatch as a cause of increased adiposity and metabolic disease later in life. Our results suggest that postnatal prevention and care should be given not only to those with low birth weight, but also to offspring of mothers having experienced adverse events during pregnancy, regardless of the size of birth (16).

Fetal growth restriction (FGR) modifications to postnatal bone metabolism and skeletal growth have been associated with low bone mass in infancy, reduced bone mass, and density in adulthood and increased risk for osteoporosis development in 
adult life, suggesting that the lack of nutrients early in life may compromise the adult skeleton (32-34). It has also been demonstrated that the fetal growth pattern affects BMC not only in small for gestational age infants, but also when birth weight is maintained within the normal range, suggesting the importance of prenatal environment in postnatal bone development (35).

Experimental studies have also demonstrated a negative long-term effect of FGR on bone size, mineral content, and strength in weaning and adult rats, speculating that FGR decreases endochondral ossification responsiveness, and in turn, postnatal linear skeletal growth, bone mineralization, and strength (36). In our study, prenatal food restriction had a significant impact on bone mineral accrual, and rats that were born to food-restricted mothers showed lower BMC and BMD values irrespective of their birth weight.

Starved rats that were born $<-2$ SD showed lower tot $\mathrm{BMC}, \mathrm{Hd} \mathrm{BMD}$, Hd BMC, Hd area whereas those born $>-2$ $\mathrm{SD}$ showed lower Abd. BMD and Abd. BMC at $26 \mathrm{~d}$ of life, although they received standard diet postnatally, than animals that were on a standard diet both pre- and postnatally. Since the nutritional postnatal environment was the same, it was prenatal food restriction that adversely affected both pathophysiological mechanisms (physical growth and bone mineral accrual) either independently or in an interaction manner.

Starved rats that were born $<-2$ SD and received standard diet postnatally showed lower tot. BMC, Hd BMC, Hd area, Abd. BMC than those born $>-2 \mathrm{SD}$, indicating that under standard postnatal nutrition, birth weight contributes significantly to bone development. Interestingly, postnatal starvation eliminated the effect of birth weight on bone development between the two prenatally starved groups. However, postnatal starvation caused significantly lower values of tot. BMC, Hd
BMD, Hd BMC, Hd area, Abd. BMD than postnatal standard diet in offspring at $26 \mathrm{~d}$ of life.

To our knowledge, this is the first experimental study in which body composition was studied in prenatally starved nutritional groups divided by birthweight in FGR and non-FGR, with subsequent postnatal food manipulation during the lactation period. The findings of the study conclude that prenatal diet critically contributes to the determination of body composition during the early postnatal stages of life, regardless of birthweight. Pediatricians might consider not only birthweight but also prenatal adverse events per se for the estimation of both the metabolic risk and possible inadequate bone accrual of infants and children, promoting adequate prevention and intervention strategies. Early detection of possible pregnancy complications (diabetes mellitus, pre-eclampsia, fetal macrosomia, FGR, etc.) (37-40) and appropriate follow-up, in addition to nutritional interventions in early infancy (breastfeeding, avoidance of overeating in order to catch-up), may program adult metabolic and bone health and ameliorate diseases with developmental origins, such as the metabolic syndrome and osteoporosis.

\section{METHODS}

This is a part of a larger study involving the effects of prenatal and postnatal food manipulation on metabolism, body composition, organ weight, and tissue morphology of the offspring. Earlier publications have emanated from this study (16). The study was initiated at the Fetal Medicine Foundation and the Harris Birthright Research Centre for Fetal Medicine, King's College Hospital, London, UK and was collaboratively conducted at the Experimental Laboratory of Aretaieion University Hospital in Athens, Greece.

\section{Rat Model of Prenatal and Postnatal Food Manipulation}

The study was approved by the Animal Research Committee of the Aretaieion University Hospital, Athens, Greece, and by the Veterinary Directorate of the Prefecture, Athens, Greece. Every effort was taken to minimize pain or discomfort in the animals.

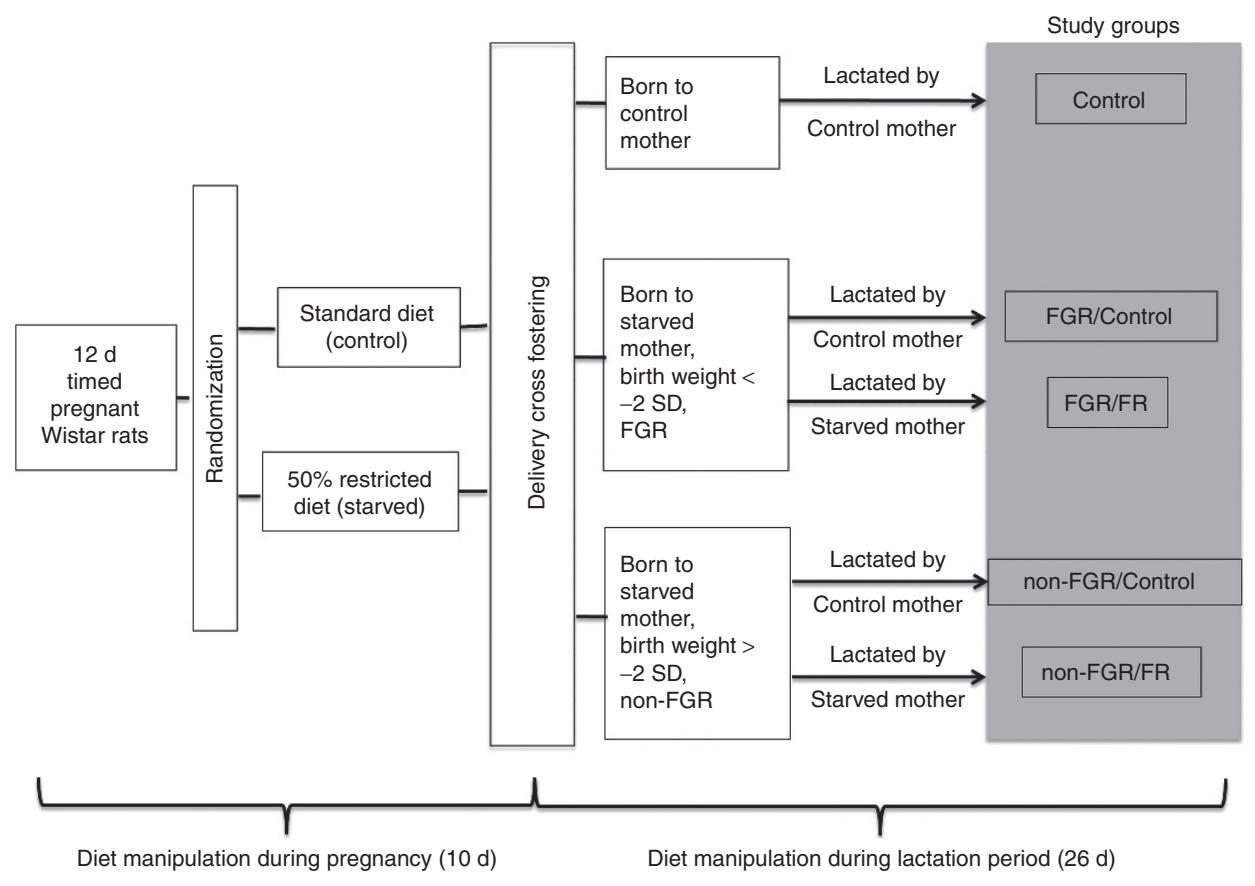

Figure 1. Experimental design of the study. 
First-time pregnant Wistar rats of the same age were obtained at 11 $\mathrm{d}$ of gestation (Harlan Laboratories B.V., Horst, The Netherlands) and housed individually in standard rat cages with free access to water. Rats were kept in the same room with constant temperature and humidity and on a controlled 12-h light to dark cycle. A model of rat dams that were either normally fed or underwent $50 \%$ food restriction during pregnancy was used.

At $12 \mathrm{~d}$ of gestation, the timed pregnant rats were randomized into one of the two following nutritional groups:

1. Control diet group: continued on an ad libitum diet of standard laboratory rodent food (4RF25, Mucedola, Milan, containing $22 \%$ protein, $3.5 \%$ fat and $50.5 \%$ carbohydrates, metabolizable energy $2,789 \mathrm{kcal} / \mathrm{kg}$ ).

2. Starved group: receiving $50 \%$ food restricted diet that was determined by quantification of normal intake in the ad libitum fed rats.

The respective diets were given from $12 \mathrm{~d}$ of pregnancy to term and throughout the 25 -d lactation period. There was no significant difference of maternal body weight between two groups.

\section{The Offspring}

Rat dams gave birth normally on day $21 ; 24 \mathrm{~h}$ after birth, the pups were culled to eight (four males and four females) per litter to normalize rearing. To differentiate the impact of prenatal food restriction and birth weight on postnatal heath, pups that were born from food restricted mothers were further divided into two subgroups:

(i) FGR group: including prenatally starved neonates with mean body weight at birth $<-2 \mathrm{SD}$ of the mean body weight of the prenatal normally fed pups.

(ii) "non-FGR" group: prenatally starved neonates with mean body weight at birth $>-2$ SD of the mean body weight of the prenatal normally fed pups.

All neonates were cross-fostered to distinguish the effects of prenatal and postnatal food manipulation and to avoid bias caused by selective maternal deprivation stress. Therefore, we cross-fostered pups so that the offspring of mothers fed on a standard diet during pregnancy were suckled by normally fed and food-restricted dams. The same cross-fostering procedure involved the offspring of food restricted mothers. Thus, the following five groups were studied: (i) normally fed prenatally/normally fed postnatally (CONTROL/CONTROL), (ii) food restricted prenatally (FGR)/ normally fed postnatally (FGR/CONTROL), (iii) food restricted prenatally (FGR)/food restricted postnatally (FGR/FR), (iv) food restricted prenatally (non-FGR)/normally fed postnatally (nonFGR /CONTROL), and (v) food restricted prenatally (non-FGR)/ food restricted postnatally (non-FGR/FR).

Litters were left undisturbed until the 25 th postnatal day. On postnatal day 26, offspring of all groups were assessed by DXA (Figure 1).

In this study, we analyzed and discussed body composition data produced by the two types of postnatal food manipulation (control diet and food restriction) on the three groups produced by prenatal and postnatal lactation nutrition assignment (CONTROL, FGR, non-FGR). We further focused on the comparison of the impact of postnatal maternal food manipulation (control diet and food restriction) on the two subgroups of prenatally food restricted animals (FGR and non-FGR).

\section{Body Composition Assessment by Dual-Energy X-Ray Absorptiometry (DXA)}

Animals were scanned using DXA (Lunar Prodigy, GE Healthcare, Diegem, Belgium). The rats were anesthetized, ventrally positioned and scanned, with spine, pelvis, femur, and tibia being the regions of interest to determine the parameters of bone mineral density $=\mathrm{BMD}$ $\left(\mathrm{g} / \mathrm{cm}^{2}\right)$, bone mineral content $=\mathrm{BMC}(\mathrm{g})$, lean mass $(\mathrm{g})$, and fat $(\%)$. Analysis was performed using the small-animal mode of the enCORE software (GE Healthcare, v. 13.40, Diegem, Belgium); the instrument was calibrated at each start.

\section{Statistical Analysis}

Continuous variables are presented with mean and SD. For the comparison of DXA variables between the study groups ANOVA were used. In case of multiple comparisons, Bonferroni correction was used to control for type I errors. Log transformations were performed on non-normal variables. To investigate the independent effect of group (postnatal maternal food manipulation), FGR (prenatal events), and sex on DXA variables, multiple linear regression analyses were performed. Also, significant interactions were tested via regression analysis. Regression coefficients and SEs were computed from the results of the linear regression analyses. All $P$ values reported are two-tailed. Statistical significance was set at 0.05 and analyses were conducted using SPSS statistical software (IBM SPSS Statistics for Windows, Version 19.0. Armonk, NY: IBM).

\section{ACKNOWLEDGMENT}

We would like to express our gratitude and deep appreciation to E. Dousi and L. Konaris for expert technical assistance during the experiments.

\section{STATEMENT OF FINANCIAL SUPPORT}

This work was supported by grant from the Fetal Medicine Foundation (https://fetalmedicine.org), UK.

Disclosure: The authors have nothing to declare. There is no conflict of Interest. None of the authors have relevant financial interests in this manuscript and we certify that no commercial financial support has been provided for this work.

\section{REFERENCES}

1. McMillen IC, Robinson JS. Developmental origins of the metabolic syndrome: prediction, plasticity, and programming. Physiol Rev 2005;85: 571-633.

2. Morrison JL, Duffield JA, Muhlhausler BS, Gentili S, McMillen IC. Fetal growth restriction, catch-up growth and the early origins of insulin resistance and visceral obesity. Pediatr Nephrol 2010;25:669-77.

3. Zhang S, Rattanatray L, Morrison JL, Nicholas LM, Lie S, McMillen IC. Maternal obesity and the early origins of childhood obesity: weighing up the benefits and costs of maternal weight loss in the periconceptional period for the offspring. Exp Diabetes Res 2011;2011:585749.

4. Amato MC, Guarnotta V, Giordano C. Body composition assessment for the definition of cardiometabolic risk. J Endocrinol Invest 2013;36:537-43.

5. Pervanidou P, Chrousos GP. Metabolic consequences of stress during childhood and adolescence. Metabolism 2012;61:611-9.

6. Eleftheriades M, Creatsas G, Nicolaides K. Fetal growth restriction and postnatal development. Ann N Y Acad Sci 2006;1092:319-30.

7. Ohta H, Kuroda T, Onoe Y, et al. Familial correlation of bone mineral density, birth data and lifestyle factors among adolescent daughters, mothers and grandmothers. J Bone Miner Metab 2010;28:690-5.

8. El Hage R, Moussa E, Hammoud A, Dandachi G, Jacob C. Birth weight is an independent determinant of whole body bone mineral content and bone mineral density in a group of Lebanese adolescent girls. J Bone Miner Metab 2010;28:360-3.

9. Lehmann AE, Ennis K, Georgieff MK, Rao R, Tran PV. Evidence for a hyporesponsive limbic-hypothalamic-pituitary-adrenal axis following early-life repetitive hypoglycemia in adult male rats. Am J Physiol Regul Integr Comp Physiol 2011;301:R484-90.

10. Dudley KJ, Sloboda DM, Connor KL, Beltrand J, Vickers MH. Offspring of mothers fed a high fat diet display hepatic cell cycle inhibition and associated changes in gene expression and DNA methylation. PLoS One 2011;6:e21662.

11. Sebert S, Sharkey D, Budge H, Symonds ME. The early programming of metabolic health: is epigenetic setting the missing link? Am J Clin Nutr 2011;94(6 Suppl):1953S-8S.

12. Lukaszewski MA, Mayeur S, Fajardy I, et al. Maternal prenatal undernutrition programs adipose tissue gene expression in adult male rat offspring under high-fat diet. Am J Physiol Endocrinol Metab 2011;301:E548-59.

13. Pervanidou P, Chrousos GP. Stress and behavior: the role of nutrients with emphasis on omega-3 fatty acids. World Rev Nutr Diet 2011;102:44-52. 
14. Bouroutzoglou M, Malamitsi-Puchner A, Boutsikou M, et al. Biochemical markers of bone resorption are present in human milk: implications for maternal and neonatal bone metabolism. Acta Paediatr 2014;103:1264-9.

15. Takemori K, Kimura T, Shirasaka N, Inoue T, Masuno K, Ito H. Food restriction improves glucose and lipid metabolism through Sirtl expression: a study using a new rat model with obesity and severe hypertension. Life Sci 2011;88:1088-94.

16. Eleftheriades M, Pervanidou P, Vafaei H, et al. Metabolic profiles of adult Wistar rats in relation to prenatal and postnatal nutritional manipulation: the role of birthweight. Hormones (Athens) 2014;13:268-79.

17. Lister JP, Blatt GJ, Kemper TL, et al. Prenatal protein malnutrition alters the proportion but not numbers of parvalbumin-immunoreactive interneurons in the hippocampus of the adult Sprague-Dawley rat. Nutr Neurosci 2011;14:165-78.

18. Gluckman PD, Hanson MA, Beedle AS, Spencer HG. Predictive adaptive responses in perspective. Trends Endocrinol Metab 2008;19:109-10; author reply 112 .

19. Brommage R. Validation and calibration of DEXA body composition in mice. Am J Physiol Endocrinol Metab 2003;285:E454-9.

20. Tanvig M, Vinter CA, Jørgensen JS, et al. Anthropometrics and body composition by dual energy X-ray in children of obese women: a follow-up of a randomized controlled trial (the Lifestyle in Pregnancy and Offspring [LiPO] study). PLoS One 2014;9:e89590.

21. de Knegt VE, Carlsen EM, Bech Jensen JE, Lade Rasmussen AM, Pryds O. DXA performance in a pediatric population: precision of body composition measurements in healthy term-born infants using dual-energy X-ray absorptiometry. J Clin Densitom 2015;18:117-23.

22. Gluckman PD, Hanson MA, Spencer HG. Predictive adaptive responses and human evolution. Trends Ecol Evol 2005;20:527-33.

23. Savchev S, Figueras F, Gratacos E. Survey on the current trends in managing intrauterine growth restriction. Fetal Diagn Ther 2014;36:129-35.

24. Balkau B, Deanfield JE, Després JP, et al. International Day for the Evaluation of Abdominal Obesity (IDEA): a study of waist circumference, cardiovascular disease, and diabetes mellitus in 168,000 primary care patients in 63 countries. Circulation 2007;116:1942-51.

25. Borel AL, Nazare JA, Smith J, et al. Visceral, subcutaneous abdominal adiposity and liver fat content distribution in normal glucose tolerance, impaired fasting glucose and/or impaired glucose tolerance. Int J Obes (Lond) 2015;39:495-501.

26. Nazare JA, Smith JD, Borel AL, et al. Ethnic influences on the relations between abdominal subcutaneous and visceral adiposity, liver fat, and cardiometabolic risk profile: the International Study of Prediction of
Intra-Abdominal Adiposity and Its Relationship With Cardiometabolic Risk/Intra-Abdominal Adiposity. Am J Clin Nutr 2012;96:714-26.

27. He F, Rodriguez-Colon S, Fernandez-Mendoza J, et al. Abdominal obesity and metabolic syndrome burden in adolescents-Penn State Children Cohort study. J Clin Densitom 2015;18:30-6.

28. Trayhurn P, Drevon CA, Eckel J. Secreted proteins from adipose tissue and skeletal muscle - adipokines, myokines and adipose/muscle cross-talk. Arch Physiol Biochem 2011;117:47-56.

29. Wajchenberg BL. Subcutaneous and visceral adipose tissue: their relation to the metabolic syndrome. Endocr Rev 2000;21:697-738.

30. Gishti O, Gaillard R, Manniesing R, et al. Fetal and infant growth patterns associated with total and abdominal fat distribution in school-age children. J Clin Endocrinol Metab 2014;99:2557-66.

31. Chen W, Wilson JL, Khaksari M, Cowley MA, Enriori PJ. Abdominal fat analyzed by DEXA scan reflects visceral body fat and improves the phenotype description and the assessment of metabolic risk in mice. Am J Physiol Endocrinol Metab 2012;303:E635-43.

32. Cooper C, Javaid MK, Taylor P, Walker-Bone K, Dennison E, Arden N. The fetal origins of osteoporotic fracture. Calcif Tissue Int 2002;70:391-4.

33. Namgung R, Tsang RC. Bone in the pregnant mother and newborn at birth. Clin Chim Acta 2003;333:1-11.

34. Briana DD, Boutsikou M, Boutsikou T, Malamitsi-Puchner A. Associations of novel adipocytokines with bone biomarkers in intra uterine growth-restricted fetuses/neonates at term. J Matern Fetal Neonatal Med 2014;27:984-8.

35. Beltrand J, Alison M, Nicolescu R, et al. Bone mineral content at birth is determined both by birth weight and fetal growth pattern. Pediatr Res 2008;64:86-90.

36. Chen H, Miller S, Lane RH, Moyer-Mileur LJ. Intrauterine growth restriction decreases endochondral ossification and bone strength in female rats. Am J Perinatol 2013;30:261-6.

37. Eleftheriades M, Papastefanou I, Lambrinoudaki I, et al. Elevated placental growth factor concentrations at 11-14 weeks of gestation to predict gestational diabetes mellitus. Metabolism 2014;63:1419-25.

38. Papastefanou I, Souka AP, Eleftheriades M, Pilalis A, Chrelias C, Kassanos D. Predicting fetal growth deviation in parous women: combining the birth weight of the previous pregnancy and third trimester ultrasound scan. J Perinat Med 2015;43:485-92.

39. Poon LC, Nicolaides KH. Early prediction of preeclampsia. Obstet Gynecol Int 2014;2014:297397.

40. Nicolaides KH. Turning the pyramid of prenatal care. Fetal Diagn Ther 2011;29:183-96. 